\title{
Espace public et production de l'information
}

The Public Sphere and the Production of Information

Isabelle Garcin-Marrou

\section{(2) OpenEdition}

Journals

Édition électronique

URL : http://journals.openedition.org/edc/7615

DOI : 10.4000/edc.7615

ISSN : 2101-0366

Éditeur

Université de Lille

Édition imprimée

Date de publication : 1 juin 2018

Pagination : $129-146$

ISBN : 978-2-917562-19-2

ISSN : $1270-6841$

Référence électronique

Isabelle Garcin-Marrou, «Espace public et production de l'information », Études de communication [En ligne], 50 | 2018, mis en ligne le 01 juin 2020, consulté le 03 janvier 2020. URL : http://

journals.openedition.org/edc/7615; DOI : 10.4000/edc.7615

(ㄷ) Tous droits réservés 


\section{Espace public et production} de l'information

The Public Sphere and the Production of Information 
La présente contribution, consacrée au thème "Espace public et production de l'information ", prend appui sur les dossiers d'Études de Communication qui ont participé à la construction d'un corpus de connaissances en sciences de l'information et de la communication. Nous suivons trois fils d'Ariane transversaux à ces dossiers : " médias et production de l'information dans l'espace public "; "productions info-communicationnelles, territoires et espaces publics »; " production de l'information et espaces publics déterritorialisés ". Nous montrons comment ces recherches fondatrices ont produit des étincelles heuristiques qui se retrouvent dans les recherches contemporaines.

Mots-clés : espace public, information, communication, territoires, débats.
This article analyzes the thematic issues of Études de Communication which participated in the construction of a corpus of knowledge in the field of Information and Communication sciences. We pursue three common threads in these issues: "media and information production in the public sphere"; "info-communicational production, territories and public spaces"; and "information production and deterritorialized public spaces". We show how these landmark studies provided a heuristic stimulus, the effects of which can be found in current research.

Keywords: public sphere, information, communication, territories, debates. 


\section{Introduction}

La présente contribution, consacrée au thème Espace public et production de l'information, prend appui sur les neuf dossiers qui ont constitué des « jalons » de recherche sur cette thématique et qui ont participé à la construction d'un corpus de connaissances en sciences de l'information et de la communication.

La relecture de ces neuf dossiers d'Études de Communication (EDC) permet de structurer la présente contribution en suivant trois fils d'Ariane transversaux. Ces fils s'articulent comme suit :

- médias et production de l'information dans l'espace public (EDC n 15 , $38,44)$;

- productions info-communicationnelles, territoires et espaces publics (EDC n 17, 18, 37) ;

- production de l'information et espaces publics déterritorialisés (EDC $\left.n^{\circ} 22,47,48\right)$.

Pour chacun de ces fils et dans chacune des trois parties qui leur sont spécifiquement consacrées, nous proposons de revenir sur les éléments fondateurs et les réflexions qui, à la relecture, produisent toujours des étincelles heuristiques ${ }^{1}$. Nous tentons ensuite de montrer comment ces étincelles se retrouvent dans le feu de recherches contemporaines. Enfin nous proposons, à nouveau pour chacun des fils d'Ariane, de spécifier en quoi les sciences de l'information et de la communication peuvent produire une épistémologie aussi féconde qu'ouverte à d'autres approches.

1.

\section{Médias et production de l'information dans l'espace public}

Une première thématique de cette contribution regroupe donc les dossiers de la revue qui ont interrogé le rôle des médias du point de vue de pratiques communicationnelles situées, d'appropriations sociales de ces pratiques et, plus largement, de ce que les sociétés contemporaines font des contenus de la production informationnelle.

1 Ce texte a été discuté, au téléphone, avec Jean-François Tétu, alors que j'attendais, dans une gare de province, des trains qui ne circulaient pas. Les questions d'information, d'espace(s) public(s) fragmentés, de territorialisation et de déterritorialisation, mots clefs de la contribution, peuvent donc aussi être constitutives des conditions de production de la recherche. Que Jean-François Tétu reçoive ici toute ma gratitude pour son œil et son esprit aussi bienveillants que précis. 
Depuis les travaux interrogeant l'arrivée de l'information internationale en continu et les redéfinitions des rapports que les sociétés entretiennent à I'information ("L'Adieu à Théophraste », formulé si élégamment par Yves Lavoinne en 1994 (Lavoinne, 15, 7-12)), les dossiers d'Études de communication ont abordé à plusieurs reprises les transformations des processus de production de l'information dans les espaces publics. Mettre en perspective les travaux portant sur les médias et la production de l'information dans l'espace public permet de comprendre comment l'attention des chercheur.es s'est modifiée dans la prise en compte des pratiques, des acteurs, et de ce qui a permis - et permet toujours - de penser le passage d'une " civilisation du journal » à une " civilisation d'internet ".

Les questions qui ont structuré les travaux sur le rôle des médias dans la production de l'information dans l'espace public peuvent être regroupées sous plusieurs angles, ici rappelés dans une tentative classificatoire non exhaustive.

Tout d'abord il faut noter cette interrogation de la (des) temporalité(s) informationnelles. Jean-François Tétu s'interroge, dès 1994, sur la " maîtrise du temps " induite par la logique de flux de la radio. Et sa conclusion ouvre à une interprétation qui peut être actualisée dans l'analyse des productions informationnelles contemporaines, fondées sur la mise en scène du « temps réel ». Ainsi J.-F. Tétu écrit-il :

«En d'autres lieux déjà, nous avons tenté de montrer, à partir de la presse écrite surtout, que l'information, dans les médias, n'était pas dirigée vers l'explication de l'événement, mais vers son attente. Cette réflexion sur la radio renforce notre hypothèse. De tous les médias, la radio, dont on a vu d'abord de quelle façon elle cherche à accompagner le temps de l'auditeur, cherche à maîtriser le temps par un primat absolu du récit sous la forme la plus élémentaire [...] le bref instant où le présentateur radio retient son souffle, sur le tir d'un pénalty, est un trou par où l'auditeur attentif peut percevoir que le temps, dont le récit construit la représentation, est entre les mains, ou plutôt dans la voix, du 'speaker' » (Tétu, 15, 88-89).

Ce « trou » par lequel l'auditeur perçoit que le temps est aux mains du journaliste n'est-il pas fortement réactualisé par la dynamique temporelle fondatrice de la production d'information contemporaine, par les médias en ligne comme par les réseaux sociaux ? Le traitement médiatique des attentats de 2015, notamment, en France, peut être envisagé sous l'angle de cette temporalité suspendue, où le public attend, avec le journaliste, que le récit reprenne.

Autre ensemble de questions, celles liées au public des médias dans l'espace public démocratique. Les médias de masse, abordés de façon fréquente dans les différentes livraisons d'EDC, évoluent en médias interactifs (web 2.0) puis voient arriver les réseaux sociaux - qui prennent une place importante dans la médiasphère contemporaine. La question des pratiques et des publics 
de ces médias, posée dès 1994, est reposée en 2015 (EDC 44). Les travaux identifient ainsi le remodelage des pratiques de réception des citoyens, qui se distancient des contenus " commerciaux ». La figure du " consommateur » est remise en question; mais si elle ne se dilue pas complètement, elle s'articule à d'autres figures, plus interactives ou, peut-être, plus citoyennes - au sens d'une reconquête de la compétence spectatorielle dans un espace public où l'alphabétisation médiatique augmente. Les figures des destinataires de l'information sont également en jeu dans cet ensemble de réflexions ; les trois âges du public, formalisés par Y. Lavoinne (Lavoinne, 15, 9) sont toujours heuristiques. Mais les figures du " citadin », du " citoyen » et du " consommateur » apparaissent également rebattues ou mêlées dans les processus médiatiques contemporains. Les trois figures ne seraient ainsi pas superposées mais relativement sécantes, dans de nombreux médias.

Les formes de l'énonciation médiatique et informationnelle sont également travaillées, et c'est peut-être là un angle de recherche pour lequel les analyses de 1994 (EDC 15) sont les plus interrogées. L'analyse des différentes livraisons d'EDC fait ainsi surgir ce constat selon lequel les rituels audiovisuels ne sont pas effacés par les nouvelles formes de contenus et pratiques médiatiques (EDC 44). Ainsi, le rôle de constitution d'un espace public par les médias semble toujours articulé à l'activité des médias généralistes - qui s'adressent à un grand public. La perspective théorique déployée dans les recherches peut être décrite comme relevant de ce que formalisait Jack Goody : l'écriture donne forme à une organisation sociale complexe et une accumulation des connaissances (Goody, 1979) ; et il apparaît fécond, comme le fait EDC 38, de s'interroger sur l'organisation sociale et l'accumulation de connaissances formée par les médias de la convergence et des techniques numériques.

Enfin, et dans un élargissement de la focale, il paraît plus que pertinent de revenir sur une contribution de Maurice Mouillaud, consacrée aux « régimes de visibilité » de l'information - aux "Lumières de la ville " comme l'écrit Y. Lavoinne. Cette réflexion demeure très féconde, si l'on songe à la façon dont M. Mouillaud décrit la «place tenue dans les médias par les banlieues (la 'violence', la 'dangerosité') [qui] est à la mesure de notre police ; id est, de l'exigence d'une société de plus en plus policée " (Mouillaud, 15, 20). Ce qui est ici questionné, c'est l'impossible transparence du discours médiatique, sa prétention impossible, quoique revendiquée, à rendre compte du réel. L'espace public se forme dans les médias d'information mais il le fait aussi par le jeu des "parts d'ombre " ${ }^{2}$, des angles morts, des recoins obscurs qui échappent à la lumière médiatique. La grande difficulté de médias nichés dans ces angles 
obscurs - comme le fut le Bondy Blog ${ }^{3}$ avant qu'il n'obtienne la place d'un média comptant dans l'écosystème médiatique général - est de faire émerger, dans l'espace public généraliste, les questions touchant aux conditions de vie des habitants des quartiers dits " périphériques " ou « difficiles " (Garcin-Marrou, 2015). Dans une autre oscillation entre ombre et lumière, le dévoilement récurrent d'informations sensibles, pour les responsables politiques, par les médias - que l'on pense aux WikiLeaks ou aux Panama Papers - ravive le rôle majeur de ces médias d'information dans le maintien d'une part d'ombre qui ne prenne pas trop de place dans les espaces publics démocratiques.

Tous ces éléments mis en perspective permettent d'ouvrir aux questions récentes qui réinterrogent le rôle - ou le magistère - des médias dans la production informationnelle de l'espace public - entendu ici au sens habermassien du terme, i.e., au sens de communauté symbolique structurée par les discours. Dans cette logique communicationnelle, la production généralisée de l'information par les médias traditionnels, par les pureplayers, dont certains s'organisent autour de l'impératif de l'enquête longue, et par les médias contributifs bouscule la place centrale qu'avaient acquise les médias de masse - depuis l'apparition, notamment, de la grande presse industrielle. Les acteurs médiatiques se démultiplient et leurs contributions respectives à la formation de l'espace public paraissent à la fois plus vivaces mais, également, plus difficiles à analyser (voire, même, simplement, à quantifier). Les figures du " citadin ", du " citoyen » et du " consommateur " sont ainsi convoquées en même temps, dans un foisonnement de supports qui interrogent le rôle des médias dans la structuration de l'espace public. En effet, et de façon paradoxale, alors que les contributions démultipliées de médias semblent pouvoir majorer le rôle de la production d'information dans la formation de l'espace public, celui-ci semble maintenir de plus en plus difficilement ses logiques d'inclusion - nous y revenons en infra. Le rôle des réseaux numériques a ainsi pu être spécifiquement questionné, sous l'angle de la réduction de la pluralité de l'information (Rebillard, 2012).

Ce qui est en jeu dans ces travaux et dans cette mise en perspective, ce sont, finalement, les formes sociales et politiques que produisent les processus info-communicationnels - allant de la production d'une "nouvelle » à la for-

3 Le Bondy Blog est né de l'initiative de Serge Michel, journaliste suisse du magazine $L ' H e b d o$, qui prit ses quartiers avec une quinzaine de collègues en relai, à Bondy, en novembre 2005, lorsque les villes des banlieues françaises s'embrasèrent à la suite de la mort de Zyed Benna et Bouna Traoré, le 27 octobre 2005, dans un transformateur EDF. L'absence quasi totale de présence des médias français dans les quartiers en révolte était apparue comme une faille informationnelle majeure au journaliste de l'Hebdo qui passa le relai, après les événements, en 2006, à une équipe de journalistes issus des quartiers dits «périphériques ». Le Bondy Blog est maintenant un média reconnu, toujours engagé dans la défense et la promotion de quartiers encore trop souvent (mal)traités par les grands médias généralistes : http://www.bondyblog. $\mathrm{fr} /$ 
mation d'une conscience individuelle et collective du monde tel qu'il s'institue par et dans les médias. La question de l'espace public et de la production de I'information touche ici à ce qui constitue le cœur de l'appartenance démocratique : la possibilité pour chacun.e, pris dans des appartenances et des pratiques, d'être un.e citoyen.ne informé.e et relié.e à l'ensemble socio-politique. Sous ce point de vue, le passage d'une civilisation du journal à une civilisation d'internet, s'il est vérifiable par (presque) chacun.e dans (presque) toutes ses activités quotidiennes, n'est ni le synonyme d'une émancipation générale ni celui d'un assujettissement aux logiques de profit qui caractériseraient la structure médiatique convergente (résultant de la convergence).

L'originalité et la force de la perspective déployée par les sciences de l'information et de la communication tient à ce que les travaux ne sont pas stricto sensu issus de l'histoire ou de la linguistique - disciplines d'origine de certains des contributeurs du numéro 15 de la revue EDC, mais qu'ils héritent de ces épistémologies et s'en ressaisissent à partir d'une approche spécifique, qui fait de l'activité communicationnelle le cœur de la machine démocratique. Outre la séparation des pouvoirs, la démocratie se fonde sur l'appartenance active de ses sujets (par le vote notamment) et celle-ci ne peut s'effectuer que dans la saisie, par les membres de l'ensemble socio-politique, des procédures de participation. Et cette dynamique démocratique, fondée sur une activité de plus en plus vivace des médias, ne s'est pas démentie depuis l'apparition de la presse ; elle demeure cependant à interroger constamment, pour que la part d'ombre de l'exercice du pouvoir ne devienne pas une entrave à la possibilité des médias à contribuer à l'enlightment des citoyen.ne.s.

\section{2.}

\section{Productions info-communicationnelles, territoires et espaces publics}

Comme une déclinaison territorialisée de la première thématique, la deuxième regroupe les dossiers qui ont interrogé les logiques médiatiques sous le point de vue de leur production située dans des espaces spécifiques. Des espaces publics régionaux au " travail territorial des médias ", en passant les espaces publics micro et ordinaires, les trois dossiers de la revue $(17,18$ et 37$)$ ont abordé les processus de la production de l'information médiatique en lien avec des configurations resserrées de l'espace public. Des espaces publics locaux sont saisis à partir des modalités de construction de leurs identités, de leurs mémoires, de leurs dynamiques culturelles, sociales et politiques.

L'analyse de ces trois dossiers permet de resituer les questions toujours vives de la production symbolique des territoires et des collectifs, du rôle de l'information et de la communication dans des "faire société " situés et des dynamiques d'appropriation, parfois conflictuelles, des productions informa- 
tionnelles et communicationnelles par les citoyen.ne.s de ces espaces publics. Les échelles de la production de l'information sont également interrogées, notamment du point de vue de la tension entre médias de masse et espaces publics locaux. Enfin, une attention est portée à la mise en œuvre des démarches comparatistes qui permettent d'identifier les décalages, les hiatus ou les convergences entre les logiques de production de l'information et du territoire et celles de leurs appropriations sociales.

Le " travail territorial » des médias constitue une perspective centrale (formalisée comme telle par le dossier 37), toujours réactualisée en fonction des productions ou formats médiatiques analysés, de la visée de mise en intelligibilité de ce que les médias font aux territoires - et réciproquement. Les territoires - souvent définis aussi par des frontières politico-administratives - sont vécus, représentés, imaginés, appropriés, traversés par des publics des médias, par des usager.e.s, par des habitant.e.s. Les travaux proposés visent ainsi à comprendre, de façon "critique ou distanciée " (Pailliart, 37, 149) comment se nouent et se jouent, dans les productions médiatiques les plus diverses - mais aussi les plus courantes (la PQR, les plateformes numériques), les définitions renouvelées de ce " qu'est » un territoire, de ce que sont sa mémoire, ses pratiques culturelles, ses formes imaginées, ses dynamiques sociales et politiques.

Comme I'indique Isabelle Pailliart en postface du dossier 37, « proposer une approche info-communicationnelle des territoires, c'est enfin éclairer la communication de l'État non seulement à partir de ses intentionnalités et de ses politiques mais également à partir de sa mise en œuvre et de la confrontation avec les pratiques sociales " (Pailliart, 37, 152). Information et communication apparaissent ainsi comme des dynamiques spécifiques de constitution d'espaces publics localisés, situés; espaces publics parcelles ou fragments d'un espace public compris selon le modèle habermassien - que les auteurs du numéro 18 entendaient, quelques années auparavant, "laïciser », et dont l'analyse permet de comprendre, au plus près des pratiques sociales, les articulations avec un espace public national et les déclinaisons locales. Les identités tissées dans ces espaces publics territorialisés et/ou localisés ne sont plus comprises sous l'angle d'une universalité (pourtant limitée à une classe ou une catégorie de citoyens dans l'espace public unifié), elles sont construites de façon proche et saisissable. La politisation de ces espaces publics partiels se fait donc sur d'autres bases, communautaires plus qu'universalistes, ou catégorielles. Mais le problème est celui de l'ombre et de la lumière : pas de focalisation sans ombre ou sans flou sur le reste. Dans l'évolution des recherches, les travaux se focalisent donc sur des lieux plus limités, ce qui permet aussi de voir les ombres de ce qui a précédé. Ce phénomène est accentué par les changements ou ruptures technologiques qui le permettent, i.e., soit le tout petit (blog ou quartier) soit le très grand (big data).

Les différentes contributions à ces trois dossiers redonnent force à l'interrogation des processus communicationnels dans des espaces plus si- 
tués, ou matérialisés (rue, campus, bureau de poste etc.) ; elles permettent ainsi de saisir, dans un grain fin, la constitution communicationnelle des collectifs qui se forment et se renouvellent dans des espaces de la vie ordinaire. Les questions centrales lancinantes et constamment reposées des identités et des communautés sont posées depuis le dossier 17 (datant de 1995) jusqu'au EDC 37 (paru en 2011). À l'échelle d'une région, d'une île, d'une ville, d'un stade, les processus de production de l'information et les modalités communicationnelles sont interrogés dans leurs modes d'institution des temps et des espaces communs; soit des espaces publics. C'est aussi dans ces dossiers que se décline la proposition faite par Bernard Miège de travailler l'espace public au pluriel (Miège, 1995), de travailler donc à comprendre la formation d'espaces publics qui peuvent être ainsi saisis dans leurs dynamiques de proximité ou de participation des habitant.e.s. Autre perspective scientifique heuristique : dans les travaux, constitutifs des livraisons 17, 18 et 37 d'EDC, la perspective info-communicationnelle se déploie dans une dimension fortement comparatiste. Les espaces publics sont abordés de façon relationnelle, les territorialisations différenciées de la production de l'information apparaissent par le jeu des contributions successives. L'effet cumulatif de ces recherches permet ainsi de dégager quelques horizons d'attente scientifiques.

Tout d'abord, la mise en perspective des différents dossiers permet de comprendre le temps scientifique plus long dans lequel s'ancrent les travaux contemporains consacrés, par exemple, à la production symbolique des territoires, reconfigurée par les réseaux sociaux. Sous ce point de vue, les travaux les plus récents mettent en œuvre ce qui était déjà décrit par I. Pailliart, à savoir une forte collaboration interdisciplinaire et la mise en œuvre de croisements méthodologiques permettant de saisir toute la complexité des espaces publics locaux - ou localisés. À cet égard, un projet de recherche comme celui intitulé "Identités numériques urbaines " - IDENUM, mené au sein du LabEx IMU 4 (auquel participent les sciences de l'information et de la communication) -, permet de comprendre comment les usages communicationnels ordinaires de la ville par utilisateurs des réseaux sociaux documentent les espaces publics de façon renouvelée. La recherche brasse des corpus complexes, faits de documents photographiques produits et diffusés par des usagers de la ville ainsi que par des acteurs collectifs (collectivités territoriales, institutions patrimoniales, associations, réseau de transport public...). La visée scientifique est de comprendre somment se fabrique une :

"Sociabilité symbolique de et dans la ville à partir de sa médiatisation numérique, abordée comme une dimension de la ville intelligente. Les réseaux sociaux sont considérés comme des dispositifs médiatiques en ce sens qu'ils déterminent les contraintes et ressources de la pratique photographique qui y est réalisée ainsi que sa gestion documentaire 
(organisation, description, partage) $»^{5}$.

De nombreux travaux collectifs, articulant sciences de l'information et informatique, par exemple, s'attachent à analyser les acteurs régionaux de l'« open data ", les villes intelligentes. Dans ces projets, les analyses permettent de comprendre les logiques info-communicationnelles, prises entre usages sociaux et techniques, entre propositions publiques et appropriations citoyennes. Dans les recherches ${ }^{6}$, ce sont des articulations de stratégies - celles des institutions, des pouvoirs locaux - et de tactiques - celles des usager.e.s des territoires qui sont passées au crible ; les territoires sont ainsi pensés comme des espaces publics en tension, dans lesquels dissensus et consensus sont constamment remis en jeu, sous la poussée, aussi, des initiatives visant à élever la capacité d'agir des habitants.

L'originalité de ces approches déployées par les sciences de l'information et de la communication est de saisir la production symbolique - sémiotique et sociale - des territoires au travers de processus spécifiquement communicationnels - distincts des regards des géographes ou des sociologues. Le matériau analysé est ainsi constitué par les pratiques des usagers de ces espaces, les inscriptions sémiotiques, les relations tissées dans des espaces « urbains " ou " de l'urbain »; et le renouvellement des recherches est ici lié à ce qui permet de " voir » ce que l'on ne voyait pas (ouverture du champ et spécialisation ou particularisation). Ce qui est en jeu ici, finalement, correspond plus à une analyse des pratiques, des tactiques - au sens de De Certeau (1990) - qui permettent à chacun.e de se reconnaître comme appartenant à un collectif territorialisé, institutionnalisé, variable selon les contours - formels ou informels - de cette territorialisation et de cette institutionnalisation qui correspondent, elles, à des stratégies.

3.

\section{Production de l'information et espaces publics déterritorialisés}

De la territorialisation à la déterritorialisation, et dans un mouvement deleuzien de pensée, nous proposons une troisième thématique articulant la production de l'information et des espaces publics. Cette thématique constitue le fil conducteur de trois dossiers d'Études de communication, parus respecti-

5 Présentation du projet IDENUM : http://imu.universite-lyon.fr/projet/idenum-identites-numeriques-urbaines/

6 La vivacité de cette perspective de recherche a été illustrée par un colloque coïncidant à l'écriture de cette contribution, et organisé à Grenoble par les collègues du Gresec sur le thème "Information - communication publique ET espaces publics sociétaux : interactions et tensions ", Université Grenoble-Alpes, 12-13 octobre 2017. 
vement en 1999, 2016 et 2017. Abordant respectivement la « médiatisation des problèmes publics » (EDC 22) puis, dans deux numéros successifs (EDC 47 et 48), les " conflictualités dans les espaces publics», ces trois livraisons de la revue envisagent les espaces publics comme des espaces communicationnels où s'identifient et se forment les problèmes publics, où se nouent les conflictualités, les rapports de force, les hégémonies et les contestations. Et depuis presque 20 ans que la revue a consacré un numéro à ces questions, le rôle des médias et des producteurs d'information dans la structuration des sociétés a continué à être interrogé, au regard des logiques constitutives d'arènes, de champs et/ou de groupes. De fait, c'est bien la production de l'information, dans la mise en sens toujours nécessaire des débats propres à l'existence de l'espace public, qui se trouve mise en questions, celles-ci étant par ailleurs renouvelées par le développement et l'appropriation ordinaire des médias sociaux, qui permettent - ou pas - un rôle nouveau de la parole ordinaire, aux côtés du magistère ancien de la parole médiatique légitime. Ces espaces publics problématiques, conflictuels, traversés de tensions, de débats, de causes, de replis ou de collectifs peuvent être saisis comme des espaces publics déterritorialisés, car, si les discours et les débats s'ancrent dans des matérialités médiatiques situées, leurs articulations s'analysent comme produisant un espace - pas forcément - idéal de parole, revivifiant la situation décrite par Jürgen Habermas (1979) comme un horizon vers lequel tendre.

Abordant la médiatisation des problèmes publics, Bernard Delforce et Jacques Noyer montrent combien la discursivité sociale est plurielle et combien doivent être prises en compte, dans la constitution des problèmes publics, les « processus de négociation et d'ajustement social pour lesquels les médias jouent un rôle structurant " (Delforce et Noyer, 22, 30). La perspective ainsi défendue par les auteurs implique de considérer la production de l'information en évitant, notamment, le médiacentrisme et en analysant comment, dans la définition de l'intérêt public, se nouent des interactions sociales, des " démarches définitionnelles » et les "difficiles ajustements " entre " cadres de définition " des problèmes publics. Un élément important de l'analyse de B. Delforce et J. Noyer tient en cette précision apportée sur le processus de problématisation publique : celle-ci n'est pas réductible au « heurt des discours [qui sont], par définition, porteurs de stratégies d'acteurs ». La «lutte des discours est à considérer comme une forme socialement construite de la lutte des acteurs "; "la publicisation d'un problème est donc aussi, du côté des acteurs - y compris les médias -, la capacité à construire dans le discours les publics visés (dont l'intérêt est convoqué par la configuration du problème) » (Delforce et Noyer, 22, 29).

Si nous mentionnons ces éléments posés dès 1999 par l'article d'ouverture du dossier, c'est qu'ils nous semblent tracer, de façon extrêmement fine, des enjeux d'analyse qui demeurent féconds - et sont actualisés par les dossiers 47 et 48 . En effet, en abordant l'espace public comme un champ de forces, la réflexion rappelle la nécessité de prendre en compte, sous un angle 
triple, les logiques de production de l'information : saisir les jeux d'acteurs, saisir la contribution - majeure - des médias dans les luttes définitionnelles et saisir les publics visés ; ces trois angles s'actualisant, notamment, dans les productions discursives. Cela implique de considérer les polyphonies discursives et, au sein de celles-ci, le processus de mise en sens de l'espace public. Pour le dire autrement, l'analyse communicationnelle de l'espace public - des espaces publics -, et des dissensus ou consensus qui le constituent, invite à considérer les lieux symboliques - ou matériels - des discussions comme des espaces où se jouent les identités des acteurs sociaux ou politiques, où se nouent leurs affrontements définitionnels, où se constituent, aussi, les publics des questions débattues.

Les pistes ouvertes par ce dossier peuvent être fortement reliées aux deux dossiers récents, qui constituent à la fois une reprise de la chaîne réflexive sur l'espace public comme lieu déterritorialisé de la construction des débats publics, mais aussi un ensemble de réflexions, très contemporaines sur les évolutions de ces formes de l'espace public communicationnel.

Les dossiers 47 et 48 abordent l'espace public sous l'angle des conflictualités, tout d'abord sous l'angle de la circulation et de la qualification des discours, puis sous l'angle des rapports sociaux et de l'hégémonie. Les titres de ces deux dossiers indiquent, pour commencer, une perspective d'analyse des espaces publics qui fait émerger des situations plus vives que celles identifiées, dans le numéro 22, relatives à la médiatisation des problèmes publics. Les deux numéros consécutifs interrogent ainsi, plus directement, les conflits dans les espaces publics. Les rapports de force sont ainsi au cœur de l'analyse communicationnelle, saisis dans leurs circulations discursives, dans leur mise en représentations et dans leurs articulations à des champs d'exercice différents. Ainsi, et dans la même veine que ce que précisaient B. Delforce et J. Noyer en 1999, Marion Dalibert, Aurélia Lamy et Nelly Quemener rappellent que les médias sont un des acteurs parmi d'autres, dans un espace public pensé à une échelle large : "l'intérêt de ce deuxième niveau à l'échelle de la sphère publique globale est qu'il invite à envisager l'articulation globale des luttes entre et dans les différentes arènes. Les grands médias deviennent alors un terrain parmi d'autres, qui ne saurait être isolé et qui doit être pensé dans ses relations aux autres terrains de lutte »(Dalibert et al., 47, 14).

Cette perspective d'analyse est extrêmement féconde en ce qu'elle permet de penser la production de l'information et les dynamiques communicationnelles dans un mouvement, continu, de re-production d'un espace public en tension(s). Comprendre l'espace public - ou les espaces publics - c'est nécessairement saisir les identités des acteurs telles qu'elles se construisent dans des ensembles de productions symboliques. Comprendre l'espace public c'est donc aussi identifier les dominations qui le structurent, les affrontements qui s'y nouent, et les ruptures de la communication qui s'y produisent, lorsque les rapports de force prennent le pas sur la possibilité d'articuler les discours. 
Comme le précisent M. Dalibert et N. Quemener, dans l'introduction du dossier qu'elles ont coordonné avec A. Lamy, "il s'agit alors d'explorer les espaces publics où se déploient des prises de position minoritaires et de saisir cette confrontation entre représentations hégémoniques et discours dissonants et/ou oppositionnels comme autant de luttes de signification " (Dalibert et al., 48, 8).

La mise en perspective de ces trois dossiers, dont deux parus quelques mois avant l'écriture de notre contribution, permet donc de rappeler la nécessité des travaux portant sur la vie des démocraties au regard de leurs processus info-communicationnels et d'articuler cette mise en perspective avec les grands débats contemporains. Le web 2.0 et ses possibilités participatives ont ainsi permis la montée en puissance de voix et de discours issus de la sphère civile, des associations ; les blogs et les réseaux sociaux ont permis la publicisation de ces discours, qui peuvent s'insérer dans les discussions sociales et politiques et battre en brèche ou renouveler la formation de la discussion publique. L'exemple de la "cause des hommes ", analysé par Stéphanie Kunert dans le dossier 48 , le montre de façon fine : "si on ne peut que difficilement connaître le nombre exact des acteurs de la 'cause des hommes', on constate sur internet une abondance de blogs d'associations, collectifs ou individus". Les discours portés par ces blogs se "sont donnés à lire et à entendre depuis plusieurs années déjà : sur internet, dans des publications de type manuels ou essais, et dans les médias de grande audience (par exemple à la télévision en ce qui concerne la cause des pères divorcés) " (Kunert, 48, 93). Or, ce que montre l'auteure, après avoir identifié la rhétorique " contre-hégémonique » de ces discours, c'est que :

"Le fait de décrire la catégorie hommes comme victime d'une inégalité structurelle dans les rapports sociaux de sexe contemporains et de présenter la 'cause des hommes' comme une cause contre-hégémonique est de l'ordre de la stratégie discursive. Mais celle-ci n'est pas sans impact sur les politiques publiques de l'égalité. En témoigne par exemple, le fait que la récente grande enquête produites sur les violences par I'INED ${ }^{7}$ ne se concentre plus sur les femmes victimes » (Kunert, 48, 105).

Dans ces processus de prise de parole et de constitution discursive de causes se jouent des luttes de signification qui ne sont plus seulement portées par les grands médias, dont l'éventail éditorial pouvait permettre la représentation homothétique d'un espace public national, unifié et unifiant. La production de l'information s'articule donc à l'activité d'instances énonciatives très hétérogènes, notamment dans leurs ressources symboliques et politiques, mais qui sont, du fait de leurs productions discursives, des acteurs constitutifs d'espaces publics fragmentaires, articulés ou opposés, et déterritorialisés.

Par ailleurs, les lieux certifiés de production de l'information - les médias - 
sont mis en concurrence avec des « non-lieux » (Augé, 1992), pour reprendre les termes de Marc Augé, dont l'identité discursive peut être fortement brouillée ou insaisissable. Le phénomène des "fake news » a ainsi contraint de grands médias généralistes à mettre sur pied des équipes de journalistes «décodeurs » chargés de "désintoxiquer », depuis leurs colonnes ou leurs sites, le débat public des fausses nouvelles ou faits alternatifs ${ }^{8}$. L'information apparaît ainsi plus menacée qu'elle ne l'avait jamais été d'effondrement et/ou d'assimilation à un bruit cybernétique, potentiellement désastreux pour les dynamiques démocratiques. À cet égard, les très nombreux contenus médiatiques parus (dans les médias généralistes et sur les réseaux sociaux), consacrés à l'immixtion de la Russie dans l'élection états-unienne de 2016, témoignent de la crainte démocratique face au brouillage des instances énonciatives. Les espaces publics voient leurs contours dilués dans des flux informationnels impossibles à tracer ; la déterritorialisation de la production de l'information alimente un soupçon extrêmement vivace envers l'activité médiatique pourtant au cœur des espaces publics. Parallèlement à cela, des acteurs en opposition aux formes démocratiques s'instituent comme instances de productions d'information "sauvages ", qui échappent au consensus social et politique : les vidéos de propagande terroriste ou la propagande de Daesh atteignent les espaces publics - ou tout du moins, une partie de ceux-ci - et apparaissent comme constitutifs de discours aussi efficaces vis-à-vis de publics qu'opposés aux formes démocratiques. Face à ces discours, les médias, producteurs d'une information ancrée dans la forme démocratique, apparaissent désarmés et impuissants ou, plus exactement, ne s'adressant pas - ou plus - au même public. Nombreux sont les processus de production d'une " information » qui remettent en jeu la cohérence et/ou les dynamiques des espaces publics contemporains. Des sphères communicationnelles se forment, qui se revendiquent ou se nouent, intentionnellement en désarticulation complète avec la sphère communicationnelle formant un espace public généraliste. La "fachosphère » ou la " djihadosphère », par exemple, rassemblent des publics, dont il est difficile de cerner les ancrages sociaux et politiques, mais dont les productions discursives surgissent, ponctuellement, dans la sphère communicationnelle des espaces publics contemporains, en en bousculant les repères, les rationalités et les mécanismes de débat. Les acteurs sont en réseaux, les débats peuvent se construire en parallèle, les arguments ne visent pas à convaincre mais à vaincre, et dans cette production déterritorialisée de l'information, la production des espaces publics semble perdre de son efficace et de sa capacité à inclure l'ensemble des sujets du politique.

Face cette démultiplication des espaces symboliques, les recherches

8 Les Décodeurs du Monde, http://www.lemonde.fr/les-decodeurs/, dont le slogan est "Venons-en aux faits "; Libération et la rubrique "Désintox », qui se présente comme la "rubrique de fact-checking [qui] détecte et corrige les erreurs du débat public ", http://www.liberation.fr/desintox/2017/03/27/desintox-qui-sommes-nous_1558052 
en sciences de l'information et de la communication peuvent contribuer à saisir comment se configurent les formes démocratiques contemporaines. Parce qu'elles envisagent, de façon conjointe, les logiques et les identités des acteurs, leurs productions symboliques et les publics que celles-ci sont susceptibles de constituer, les analyses info-communicationnelles proposent d'éclairer les configurations démocratiques telles que produites par des paysages médiatiques extrêmement divers, denses et évolutifs. Le chantier est immense, car les matériaux sont infinis. Ils supposent des méthodologies d'analyse quantitative de données massives et des processus permettant de déployer, aussi, des analyses qualitatives restituant les constructions sociales et symboliques des espaces publics. Mais, dans leur histoire relativement jeune et pourtant déjà riche, les sciences de l'information et de la communication se sont outillées pour ces recherches complexes et voient émerger des collectifs de chercheur.e.s qui mettent en œuvre des analyses qu'un.e chercheur.e ne pourrait plus mener seul.e.

\section{Pour conclure... provisoirement}

Difficile de conclure après avoir tenté de montrer combien la thématique "Espace public et production de l'information » était vivace et constamment réactualisée. Pour clore notre propos, peut-être cependant un mot est-il nécessaire pour signaler que les trois fils d'Ariane ici travaillés ont inversé l'ordre des termes proposés pour la thématique générale : les médias et les logiques de production info-communicationnelles conduisant à envisager la contribution à la construction d'un espace public ou d'espaces publics. Deux remarques peuvent être faites en conclusion. La première est que l'analyse des pratiques et des contenus info-communicationnels constitue, selon nous, ce par quoi la compréhension des logiques sociales et politiques est possible. Si les analyses doivent rester situées, précises et modestes quant à leur condition de validité plus générale, il n'en reste pas moins que la saisie d'un espace public, dans sa dynamique, ne peut se faire que par l'étude de son cœur battant, communicationnel. Les sujets du politique ne le sont que parce qu'ils peuvent en saisir - à des degrés très divers - les enjeux. La paraphrase d'un mot de Simone de Beauvoir pourrait ainsi être " on ne naît pas citoyen.ne, on le devient ", notamment par ses pratiques médiatiques, info-communicationnelles.

La seconde remarque est que le passage de la notion d'espace public au singulier - à celle d'espaces publics - au pluriel - rend finalement compte de cette profusion de pratiques, de médias, de supports d'information et de communication qui placent les sujets dans des sphères d'échange ou de confrontation extrêmement nombreuses, articulées et elles-mêmes incluses dans des dynamiques plus larges remontant - ou pas - à l'échelle d'un espace public unifié. Mais le concept d'espace public demeure central parce qu'il articule des notions ou concepts majeurs issus de diverses disciplines : signifiants, signification et énonciation pour la sémiologie ; champ, habitus et rapports 
sociaux pour la sociologie; trace et marques pour l'histoire et la géographie, etc. Les sciences de l'information et de la communication ont pour principal intérêt de mêler ces "ingrédients " conceptuels; elles mettent de l'acteur social sous ou avec les mots, et elles mettent du sens dans les rapports sociaux. Plus fondamentalement, les sciences de l'information et de la communication mettent en œuvre des concepts constitutifs de l'existence des ensembles socio-politiques : fonctions et normes, conflits et règles, signification et système. Ainsi, travailler sur "l'espace public et la production de l'information ", c'est s'obliger à la complexité et à la pensée plurielle; et ce n'est pas, là, la moindre des qualités de la discipline que d'avoir réussi à traduire cette pensée plurielle en actes de recherche. 
Bibliographie

Augé M. (1992). Non-lieux. Introduction à une anthropologie de la surmodernité, Paris, La Librairie du XXIe siècle.

Dalibert M., Lamy A., Quemener N. (2016). «Introduction ». In Études de communication, $\mathrm{n}^{\circ} 47$, p. 7-20.

Dalibert M., Lamy A., Quemener N. (2017). «Introduction ». In Études de communication, $\mathrm{n}^{\circ} 48$, p. 8-19.

De Certeau M. (1990). L'invention du quotidien. 1. arts de faire, Paris, Gallimard.

Delforce B., Noyer J. (1999). «Pour une approche interdisciplinaire des phénomènes de médiatisation : constructivisme et discursivité sociale ». In Études de communication, $\mathrm{n}^{\circ} 22$, p. 13-39.

Garcin-Marrou I. (2015). « De l'exclusion à la 'guerre'. Les émeutes de 2005 et 2010 dans la presse française ". In Carpenter J., Horvath C. (dir.), Regards croisés sur la banlieue, Bruxelles, Peter Lang Éditions, p. 91-106.

Goody J. (1979), La raison graphique. La domestication de la pensée sauvage, Paris, Les Éditions de Minuit.

Habermas J. (1988), L'Espace public Archéologie de la publicité comme dimension constitutive de la société bourgeoise, Paris, Payot.
Kunert S. (2017). « Stratégies de légitimation et configurations discursives de la 'cause des hommes' ». In Études de communication, $\mathrm{n}^{\circ} 48$, p. 91-110.

Lavoinne Y. (1994). "L'Adieu à Théophraste ". In Études de communication, $\mathrm{n}^{\circ} 15$, p. 9.

Miège B. (1995). « L'espace public : perpétué, élargi et fragmenté ". In Pailliart I. (dir.), L'espace public et l'emprise de la communication, Grenoble, Ellug, p. 163-175.

Mouillaud M. (1994). "L'information ou la part de l'ombre ». In Études de communication, $n^{\circ} 15$, p. 20.

Pailliart I. (2011). " Postface». In Études de communication, $\mathrm{n}^{\circ} 37$, p. 149.

Plenel E. (1992). La Part

d'ombre, Stock, Paris.

Rebillard F. (2012). «Présentation». In Réseaux, "Internet et pluralisme de l'information », vol. 176, p. 9-25.

Tétu J.-F. (1994). « La radio et la maîtrise du temps ". In Études de communication, $n^{\circ} 15$, p. 88-89.

\section{Références Études de communication}

Études de communication, Dossier De la Gazette à C.N.N., les gestes d'informer, n 15, 1994.

Études de communication, Dossier Médias et communication dans un espace régional, $n^{\circ} 17,1995$. 
Études de communication,

Dossier Espaces publics de la vie ordinaire, $\mathrm{n}^{\circ} 18,1996$.

Études de communication, Dossier Images de territoires et " travail territorial » des médias, n³7, 2011.

Études de communication, Dossier L'éducation à l'information, aux TIC et aux médias : le temps de la convergence, $\mathrm{n}^{\circ} 38,2012$.

Études de communication, Dossier Pratiques télévisuelles à I'heure du numérique, $\mathrm{n}^{\circ}$ 44, 2015.

Études de communication, Dossier La médiatisation des problèmes publics, $n^{\circ} 22,1999$.

Études de communication, Dossier Circulation et qualification des discours. Conflictualités dans les espaces publics (1), n 47, 2016.

Études de communication, Dossier Rapports sociaux et hégémonie. Conflictualités dans les espaces publics (2), $\mathrm{n}^{\circ} 48,2017$. 